\title{
Requisição de Análises de "Rotina" em Medicina Geral e Familiar: Um Estudo Observacional Transversal Analítico Baseado na Prática em Portugal
}

\author{
"Routine" Laboratory Test Requests in Family Medicine: \\ A Cross-Sectional Study Based on Clinical Practice in \\ Portugal
}

\author{
Margarida Gil CONDE $\rrbracket^{1}$, Raquel Carmona RAMOS ${ }^{1}$, Ana RENTE ${ }^{2}$, Carina AFONSO ${ }^{3}$, Cristina Jesus HENRIQUES ${ }^{4}$, \\ Rita Grossinho REIS 5
}

Acta Med Port 2020 Oct;33(10):657-663 - https://doi.org/10.20344/amp.12653

\section{RESUMO}

Introdução: Em Portugal os utentes têm a convicção de que devem realizar análises laboratoriais mesmo na ausência de fatores de risco, nomeadamente história familiar. Estas são designadas análises de "rotina". O presente estudo pretendeu analisar o padrão de requisição de análises de "rotina" em Medicina Geral e Familiar em Portugal.

Material e Métodos: Desenvolvemos um estudo observacional transversal analítico que teve como população alvo os médicos pertencentes aos cuidados de saúde primários em Portugal. Os médicos foram questionados quanto ao seu padrão de requisição e sobre quais os motivos da requisição ou não de análises de "rotina". As questões tiveram por base as análises clínicas mais prescritas pelos médicos do Agrupamento de Centros de Saúde Lisboa Central.

Resultados: A maioria dos médicos afirmou prescrever análises de "rotina" (51,4\%). Verificou-se uma relação estatisticamente significativa entre ser especialista e a requisição de análises de "rotina" ( $p=0,013)$. As análises clínicas mais prescritas na idade adulta foram a determinação de colesterol total $(92,2 \%)$ e de glicemia. Na idade pediátrica foram a determinação de glicemia, de colesterol total e o hemograma. A maioria dos médicos $(79,4 \%)$ que prescreve análises de "rotina" fá-lo como forma de rastreio e os não prescritores não realizam análises maioritariamente $(80,8 \%)$ porque não se encontra de acordo com a evidência científica.

Discussão: No nosso estudo observámos diferenças na prática de Medicina Geral e Familiar em Portugal, nomeadamente no que diz respeito à requisição de análises de "rotina" por médicos de diferentes regiões, graus de especialização e idade. Verificámos que existe uma associação entre prescrever análises de rotina e o pedido das mesmas para realização de rastreio o que parece demonstrar que os médicos que referem prescrever análises de "rotina" têm a intenção, com as mesmas, de rastrear diversos tipos de patologia, embora os doentes não apresentem sintomatologia ou riscos que o justifiquem. A maior parte dos médicos que não prescreve análises de "rotina", não concordam com o rastreio de indivíduos assintomáticos o que está de acordo com a evidência.

Conclusão: Os nossos resultados sugerem que existe um pedido excessivo de análises de "rotina" que pode levar a sobrediagnóstico e sobretratamento sendo para isso necessária a criação de estratégias globais de marketing social para mudar a cultura vigente. Palavras-chave: Medicina Geral e Familiar; Padrões de Prática Médica; Portugal; Técnicas de Laboratório Clínico; Testes Diagnósticos de Rotina

\section{ABSTRACT}

Introduction: In Portugal, patients still believe they should perform a periodic check-up. The present study was designed to study the prescription pattern of "routine" laboratory tests in the Family practice in Portugal.

Material and Methods: We performed a cross-sectional study in the Portuguese primary healthcare setting. We surveyed physicians on their "routine" laboratory test request pattern and the reasons for requesting or not requesting laboratory tests. The questions were based on the panel of the most prescribed tests in the of Central Lisbon health centre group.

Results: Most of the inquired doctors said they requested "routine" laboratory tests $(51.4 \%)$. There is a significant difference in the request of "routine" laboratory tests performed by trainees or specialists $(p=0.013)$. The most requested laboratory tests in adults are total cholesterol $(92.2 \%)$ and blood glucose while the most requested laboratory tests in children are blood glucose, total cholesterol and full blood count. Many doctors $(79.4 \%)$ that request "routine" laboratory tests do so to perform screening and the doctors that do not request routine" laboratory tests do so mainly $(80.8 \%)$ because there's lack of scientific evidence.

Discussion: We found differences in the prescription pattern of Family Physicians in Portugal, namely regarding the request of "routine" laboratory tests by doctors from different regions, degrees of specialization and age. We found that there is an association between prescribing "routine" laboratory tests and their request for screening. These physicians aparently want to track different types of pathology, even though patients have no symptoms or risks that could justify it. Most doctors, who do not prescribe "routine" laboratory tests, do not agree with screening for asymptomatic individuals, which is consistent with the evidence.

Conclusion: Our results suggest that there is an excessive request of laboratory tests which can lead to overdiagnosis and overtreatment that requires global Social Marketing strategies to change the prevailing culture.

1. Unidade de Saúde Familiar Vasco da Gama, Agrupamentos de Centros de Saúde Lisboa Central. Administração Regional de Saúde de Lisboa e Vale do Tejo. Lisboa. Portugal.

2. Unidade de Cuidados Saúde Personalizados Olivais. Agrupamentos de Centros de Saúde Lisboa Central. Administração Regional de Saúde de Lisboa e Vale do Tejo. Lisboa. Portugal.

3. Unidade de Saúde Familiar Artemisa. Agrupamentos de Centros de Saúde Cascais. Administração Regional de Saúde de Lisboa e Vale do Tejo. Lisboa. Portugal.

4. Unidade de Saúde Familiar Sétima Colina. Agrupamentos de Centros de Saúde Lisboa Central. Administração Regional de Saúde de Lisboa e Vale do Tejo. Lisboa. Portugal.

5. Unidade de Saúde Familiar Santa Maria. Agrupamentos de Centros de Saúde Médio Tejo. Administração Regional de Saúde de Lisboa e Vale do Tejo. Tomar. Portugal.

$\square$ Autor correspondente: Margarida Gil Conde. margarida.gil.conde@gmail.com

Recebido: 05 de agosto de 2019 - Aceite: 22 de novembro de 2019 | Copyright @ Ordem dos Médicos 2020 
Keywords: Clinical Laboratory Techniques; Diagnostic Tests, Routine; Family Practice; Portugal; Practice Patterns, Physicians

\section{INTRODUÇÃO}

Segundo a definição da World Organization of Family Doctors (WONCA), o médico de família (MF) exerce o seu papel profissional promovendo a saúde, prevenindo a doença e prestando cuidados curativos, de acompanhamento ou paliativos. ${ }^{1}$

A prevenção de doenças é uma componente basilar dos cuidados de saúde primários. Em parte, esta é realizada através de testes de rastreio que são procedimentos realizados numa população assintomática de forma a averiguar a presença ou não de uma determinada doença. A prevenção quaternária, ou seja, evitar testes e tratamentos desnecessários e os malefícios a eles associados, também deve ser considerada parte das práticas preventivas dos médicos de família. ${ }^{2}$

Como o nome indica, análises de "rotina" (AR) implicam uma índole conservadora e de realização de exames de forma regular a populações de diferentes faixas etárias. $\mathrm{O}$ termo refere análises de sangue e urina realizadas a um indivíduo assintomático ${ }^{3}$ e que deverão ser repetidas num espaço de tempo determinado, com o propósito de realizar um diagnóstico precoce, de prevenir a doença e para tranquilizar o doente.

O sobrediagnóstico é um problema que tem vindo a crescer em todo o Mundo, ${ }^{4}$ é supérfluo e acarreta prejuízo no sentido em que culmina com stress psicológico e ainda, testes de diagnóstico, medicação e tratamentos desneces-

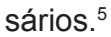

Em Portugal a maioria dos adultos considera necessário a realização quase anual de um grande número de exames médicos e a maior parte indica que estes lhes são prescritos. ${ }^{6} \mathrm{Na}$ prática de Medicina Geral e Familiar (MGF), continua a não se verificar consistência entre médicos relativamente à avaliação complementar requerida para efetuar um controlo de "rotina".

Vários estudos indicam que os profissionais de saúde, apesar das recomendações baseadas na evidência, continuam a tomar decisões na prática clínica cuja eficácia não está provada. ${ }^{7} \mathrm{~A}$ revisão mais recente deste tema identificou 14 estudos aleatorizados, com um total de 182880 participantes, em que se compararam outcomes associados a prescrição ou não de AR. Não se verificou efeito desta diferença no risco de morte, quer por doenças cardiovasculares quer por neoplasias. ${ }^{3}$

Embora em MGF se dê primazia às decisões baseadas no princípio da individualidade, em que a prática deve ser adequada a cada doente, existem padrões de evidência que remetem a favor ou contra a realização de determinada avaliação analítica devendo ser respeitados os estudos mais atuais. ${ }^{8}$

A Canadian Task Force on the Periodic Health Examination e a US Preventive Services Task Force, recomendam poucas $\mathrm{AR}^{9}{ }^{9}$ Estas análises são sem dúvida ferramentas diagnósticas importantes, essenciais no decurso da investigação e gestão de muitas doenças agudas e crónicas.
Contudo, o pedido excessivo de análises pode servir de conforto psicológico para o médico, que deposita maior confiança nos exames complementares de diagnóstico do que na observação clínica, ${ }^{10}$ o que poderá criar falsa segurança.

Neste sentido, na última década, em Portugal têm surgido diversas normas de orientação clínica disponibilizadas, nomeadamente, pela Direção-Geral da Saúde (DGS) para a gestão de requisição de meios complementares de diagnóstico, como é o caso da norma "Prescrição e determinação do hemograma" 11 e da norma "Indicações para prescrição do ionograma". ${ }^{2}$

Apesar desta medida, em Portugal não existem estudos que caracterizem a requisição de AR em MGF, o que motivou a investigação nesta área.

Assim, este estudo teve como objetivo principal analisar o padrão auto-reportado de requisição de AR na prática clínica da MGF em Portugal. Pretendeu-se ainda, como objetivos secundários, verificar em que é que os médicos se baseiam para a requisição de AR e se existe divergência nas análises pedidas entre profissionais de diferentes regiões do país, sexo, idade ou grau de especialização.

\section{MATERIAL E MÉTODOS}

Desenhou-se um estudo observacional transversal analítico, cuja população-alvo teve como critérios de inclusão médicos especialistas em MGF e internos de terceiro e quarto ano desta especialidade a trabalhar no Serviço Nacional de Saúde quer em Portugal continental quer nas regiões autónomas dos Açores e Madeira. Os critérios de exclusão definidos contemplaram os internos da especialidade de MGF do primeiro e segundo ano, por considerarmos não terem ainda autonomia na requisição de meios complementares de diagnóstico (MCDTs).

Foi calculada a dimensão da amostra para um universo estimado de 6220 médicos. Este valor teve por base os valores fornecidos pela Administração Central do Sistema de Saúde (ACSS), no final do ano de 2016, de 906 internos de MGF que se encontram no terceiro e quarto ano da especialidade e 5314 especialistas de MGF. O tamanho da amostra calculado, através dos sistemas online OpenEpi ${ }^{\circledR}$, foi de 362 participantes, para uma margem de erro de $5 \%$ e um intervalo de confiança de $95 \%$.

A recolha de dados foi realizada através da aplicação de um questionário online elaborado pelas investigadoras principais, que foi divulgado através de correio eletrónico, por todas as Administrações Regionais de Saúde (ARS) a nível nacional. O questionário apenas foi acedido após os participantes declararem consentir com os termos do estudo. O período de recrutamento decorreu entre janeiro e julho de 2017.

A primeira parte do questionário foi dirigida à caracterização do participante (especialista ou interno e local de trabalho), ao padrão de requisição de AR por parte dos 
médicos e do pedido destes exames pelos doentes. O local de trabalho foi apenas respondido por especialistas, por termos considerado que os internos têm um local de trabaIho provisório. Foi explicado aos participantes que $A R$, no contexto deste trabalho, são as análises clínicas realizadas a utentes assintomáticos e sem fatores de risco. Os médicos identificaram se prescrevem ou não AR (prescritores ou não prescritores) e quais os motivos para prescreverem ou não este tipo de análises clínicas.

A segunda parte do questionário foi orientada apenas para os médicos prescritores de AR e pretendeu identificar as análises pedidas em idade pediátrica (< 18 anos) e em idade adulta ao utente assintomático, sem antecedentes pessoais e familiares relevantes.

A terceira parte do questionário foi respondida por todos os participantes e pretendeu avaliar os motivos para o pedido individual de cada análise, tendo por base as análises mais requisitadas no Agrupamento de Centros de Saúde (ACES) de Lisboa Central (hemograma, perfil glicémico, perfil lipídico, função hepática, função renal, função tiroideia, VS, PSA).

Para a análise estatística, os dados foram inseridos no software estatístico IBM ${ }^{\circledR}$ SPSS $^{\circledR}$ Statistics 23, posteriormente validados e foi realizada a análise estatística através do mesmo software.

As variáveis quantitativas foram sumarizadas através de estatísticas descritivas nomeadamente média, mediana, desvio padrão, mínimo e máximo, e as variáveis qualitativas através de frequência absoluta $(n)$ e relativa (\%). Na análise das variáveis qualitativas utilizaram-se: o teste qui-quadrado $(Q Q)$ ou teste de exato de Fisher (quando aplicável). A comparação de dois grupos independentes face a uma variável quantitativa foi efetuada através do teste não paramétrico de Mann-Whitney. Os testes estatísticos foram efetuados bilateralmente considerando um nível de significância de 5\%.

A realização do estudo foi aprovada pelas Comissões de Ética da ARS de Lisboa e Vale do Tejo (ARSLVT) e Centro.

Tabela 1 - Caracterização demográfica (sexo, idade, grau)

\begin{tabular}{lc}
\hline & $\begin{array}{c}\text { Total } \\
(\mathrm{n}=323)\end{array}$ \\
\hline Sexo, $\mathbf{n}(\%)$ & $105(32,5)$ \\
Masculino & $218(67,5)$ \\
Feminino & \\
Idade (anos) & 38,04 \\
Média & 26 \\
Mínimo & 68 \\
Máximo & \\
Grau, $\mathbf{n}(\%)$ & $102(31,6)$ \\
Interno & $221(68,4)$ \\
Especialista & \\
\hline
\end{tabular}

\section{RESULTADOS}

O questionário foi respondido por um total de 340 médicos. Foram excluídas 17 respostas por corresponderem a médicos do primeiro e segundo anos de internato, ficando assim o total da amostra 323 .

A amostra foi constituída por $68,4 \%$ de especialistas e $31,6 \%$ de internos, sendo $67,5 \%$ do sexo feminino e $32,5 \%$ do sexo masculino. A idade dos participantes variou entre os 26 e os 68 anos (média $=38,04$ anos, DP $=11,57$ anos) (Tabela 1).

O local de trabalho foi respondido apenas por especialistas, representando 209 respostas válidas. A ARSLVT constituiu $58,4 \%$ da amostra, seguindo-se a ARS Norte com $17,2 \%$ e ARS Centro com 16,3\%. As restantes regiões: Alentejo, Algarve e Ilhas representaram 8,0\% (Tabela 2).

Não se verificou associação entre o género dos médicos e o facto de prescreverem ou não $\operatorname{AR}(p=0,337)$.

Mais de metade dos médicos ( $n=166,51,4 \%$ ) que responderam ao questionário requisitam AR. Destes, 108 $(65,1 \%)$ são do sexo feminino e $58(34,9 \%)$ são do sexo masculino. Ainda relativamente aos médicos que prescrevem $A R, 25,3 \%$ são internos, enquanto $74,7 \%$ são especialistas. Por outro lado, dos médicos que referem não prescrever AR (48,6\%), 38,2\% são internos e $61,8 \%$ são especialistas. Através do teste estatístico do qui-quadrado $(p=0,013)$ verifica-se que existe uma diferença significativa na proporção de médicos internos/especialistas entre os grupos da requisição das AR.

Um total de 311 médicos $(96,3 \%)$ referiram que os doentes pedem para prescrever AR na consulta e apenas 12 médicos $(3,7 \%)$ indicaram não receber esse pedido por parte dos doentes.

A Tabela 3 apresenta a estatística descritiva dos médicos especialistas que requisitam AR e a respetiva ARS a que pertencem. Na ARSLVT $54,0 \%$ dos médicos prescrevem AR, na ARS Centro 67,6\%, na ARS Norte 38,9\% e nas restantes regiões de saúde (Algarve, Alentejo, Ilhas) $70,6 \%$.

A Fig. 1 apresenta a distribuição das análises prescritas em idade pediátrica e adulta. Dos médicos que prescrevem $A R$, mais de $60 \%$ não prescreve análises em idade pediátrica.

Apenas 9,8\% dos internos prescritores revelaram requisitar urina II em idade pediátrica e $28,0 \%$ dos especialistas referiram o mesmo. Através do teste de Fisher $(p=0,019)$

Tabela 2 - Local de trabalho

\begin{tabular}{lc}
\hline & $\begin{array}{c}\text { Total } \\
(\mathrm{n}=221)\end{array}$ \\
\hline Local de Trabalho, $\mathbf{n}(\%)^{\mathrm{a}}$ & \\
\hline ARS LVT & $122(58,4)$ \\
ARS Centro & $34(16,3)$ \\
ARS Norte & $36(17,2)$ \\
Outras regiões & $17(80)$ \\
\hline
\end{tabular}

a: Informação recolhida apenas a especialistas

ARS: Administração Regional de Saúde; ARSLVT: Administração Regional de Saúde de Lisboa e Vale do Tejo 
Tabela 3 - Prescrição de AR e local de trabalho

\begin{tabular}{lccc}
\hline & $\begin{array}{c}\text { Prescreve } \\
(\mathrm{n}=115)\end{array}$ & $\begin{array}{c}\text { Não prescreve } \\
(\mathrm{n}=94)\end{array}$ & $\begin{array}{c}\text { Total } \\
(\mathrm{n}=209)\end{array}$ \\
\hline ARS, $\mathrm{n}(\%)$ & & & $122(100,0)$ \\
ARSLVT & $66(54,0)$ & $56(46,0)$ & $34(100,0)$ \\
ARS Centro & $23(67,6)$ & $11(32,4)$ & $36(100,0)$ \\
ARS Norte & $14(38,9)$ & $22(61,1)$ & $17(100,0)$ \\
Outras Regiões & $12(70,6)$ & $5(29,4)$ & $209(100,0)$ \\
Total & $115(55,0)$ & $94(45,0)$ & \\
\hline
\end{tabular}

AR: análises de rotina; ARS: Administração Regional de Saúde; ARSLVT: Administração Regional de Saúde de Lisboa e Vale do Tejo

foi possível verificar uma diferença estatisticamente significativa na requisição de urina II em idade pediátrica entre internos e especialistas.

Revelaram prescrever urina II em idade adulta 31,7\% dos internos em comparação com $60,0 \%$ dos especialistas verificando-se, através do teste estatístico do qui-quadrado $(p=0,02)$, uma diferença estatisticamente significativa na requisição de urina II em doentes adultos entre internos e especialistas. Os resultados obtidos no teste de Fisher para a requisição de ácido úrico $(p=0,004)$ e VS $(p=0,028)$ em idade adulta indicam que também existe uma diferença significativa na requisição destas duas análises entre internos e especialistas. Quatro internos (9,8\%) referiram prescrever ácido úrico em idade adulta em comparação com 41 especialistas $(32,8 \% \%)$ e apenas um interno $(2,4 \%)$ revelou prescrever VS em idade adulta enquanto 19 especialistas $(15,2 \%)$ indicaram prescrever esta análise neste grupo etário.
Verificou-se que existe uma associação entre a requisição de AR e o pedido de cada uma das análises laboratoriais com a intenção de realizar rastreio (hemograma, perfil glicémico, urina II, perfil lipídico, função hepática, função renal, VS, função tiroideia, PSA).

Foi feito o levantamento dos motivos para requisição e não requisição de AR, apresentados nas Tabelas 4 e 5, respetivamente.

\section{DISCUSSÃO}

A maioria dos portugueses consideram que devem efetuar AR ao sangue e urina, com uma frequência média de 12 meses. ${ }^{13}$ Com este estudo verificou-se que mais de metade dos médicos ainda prescreve $A R$, o que não está de acordo com as recomendações atuais que defendem não existir benefício (mas sim um potencial aumento do risco de causar dano) para o doente na requisição de AR ${ }^{6}$ Impôs-se a necessidade de realizar um estudo qualitativo para apurar

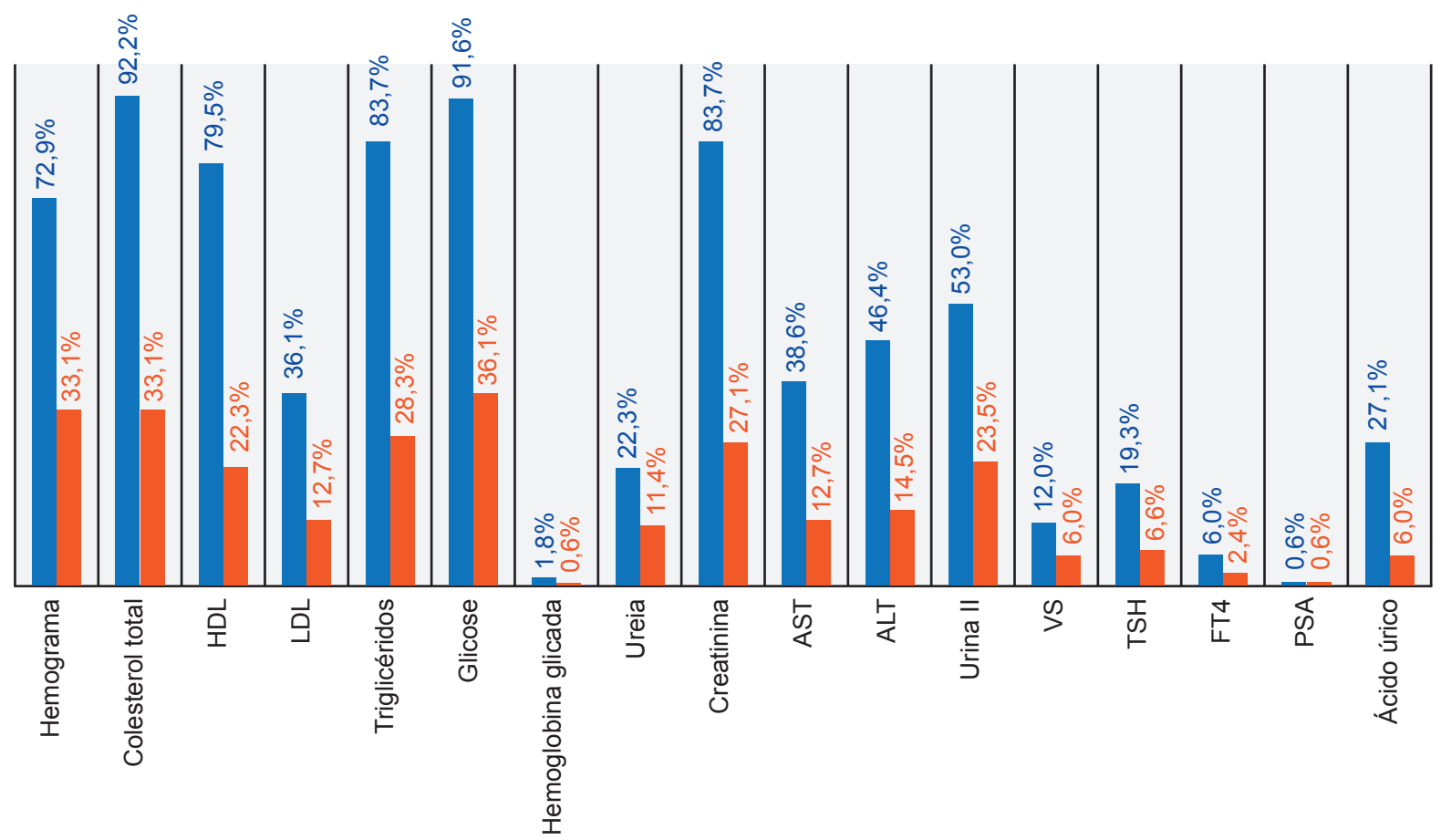

Requisição de AR a adultos (\%)

Requisição de AR a crianças (\%)

Figura 1 - Gráfico de barras das AR requisitadas a adultos e a crianças.

AR: análises de rotina; AST: aspartato aminotransferase; ALT: alanina aminotransferase; TSH: hormona estimuladora da tiróide; fT4: tiroxina; PSA; antigénio específico da próstata 
os motivos que levam à manutenção desta má prática nomeadamente explorar se existe uma perversão da relação médico-doente na qual o médico não tenha poder para fazer face a questões culturalmente instituídas.

Mostrou-se também que grande parte dos médicos prescritores de AR são especialistas, o que se reflete no facto da média das idades dos médicos não prescritores ser 10 anos menor que a dos médicos prescritores.

Podemos constatar que existe uma associação entre ser especialista ou interno e a requisição de $A R$, sendo que os especialistas prescrevem mais AR. Outros estudos são necessários para compreender o motivo desta diferença que, poderá ou não, dever-se a discrepâncias na formação dos diferentes profissionais, ou influência da experiência prática nos padrões de requisição.

Não podemos deixar de considerar a possibilidade de ter havido respostas concordantes com as normas de requisição, mas que não refletem a prática, visto serem auto reportadas. A percentagem elevada de médicos que referem não prescrever AR poderá ser um reflexo deste efeito.

Relativamente ao local de trabalho verificou-se que na região centro existem mais prescritores que não prescritores, na ARSLVT o número de prescritores é bastante semelhante ao de não prescritores, sendo a região norte onde se verifica o menor número de médicos prescritores. Tendo em conta o facto de a amostra ser pouco representativa de algumas regiões, verificando-se clara prevalência de respostas de médicos da região de Lisboa e Vale do Tejo, mais estudos são necessários para descrever melhor o padrão de requisição com base na região e compreender o porquê desta diferença regional.

Não existem muitos estudos sobre a pertinência do pedido de análises em idade pediátrica sendo justificada apenas em alguns casos específicos, ${ }^{11}$ mas não em indivíduos assintomáticos e sem fatores de risco, o que foi corroborado pela maioria dos médicos prescritores de análises, visto que mais de $60 \%$ não prescreve em idade pediátrica.

Das análises estudadas verificou-se uma maior incidên- cia na requisição de Colesterol e glicemia, talvez este facto possa ser justificado pela maior prevalência de patologias como dislipidemia, doença cardiovascular e diabetes mellitus. ${ }^{14,-16}$

Como se verificou no estudo Preventive health services implemented by family physicians in Portugal, os Médicos de Família portugueses têm uma alta concordância com as recomendações da USPSTF para a prática de medicina preventiva, ${ }^{17}$ o que pode ter influenciado a escolha de AR que estudam a glicemia e o perfil lipídico, embora, de acordo com as recomendações, estas se apliquem apenas a doentes com fatores de risco e não a utentes assintomáticos, sem fatores de risco.

A requisição de urina tipo II não é recomendada nos vários estudos e orientações clínicas sobre a pertinência do pedido desta análise, sendo consensual que só em situações específicas é que o pedido é considerado benéfico para o utente. ${ }^{17,18} \mathrm{~A}$ requisição desta análise (urina II) é efetuada mais frequentemente por especialistas que por internos, o que também se verifica na requisição de VS e ácido úrico, levando-nos a concluir que não só existem diferenças estatisticamente significativas, entre especialistas e internos, no facto de pedir ou não AR como também no tipo de análises requisitadas.

Verificou-se que existe uma associação entre prescrever AR e o pedido de AR para realização de rastreio (hemograma, perfil glicémico, urina II, perfil lipídico, função hepática, função renal, velocidade de sedimentação, função tiroideia, PSA). Esta associação parece demonstrar que os médicos que referem prescrever AR têm a intenção, com as mesmas, de rastrear diversos tipos de patologia, embora os doentes não apresentem sintomatologia ou riscos que o justifiquem.

Podemos concluir que a maior parte dos médicos que não prescreve $A R$, não concordam com o rastreio de indivíduos assintomáticos através da requisição das análises incluídas neste estudo. Esta prática está de acordo com a evidência científica,,$^{9,19}$ tendo sido esse o principal motivo

Tabela 4 - Motivos para prescrição de AR

\begin{tabular}{lrrr}
\hline & Sim & Não & Total \\
\hline Motivo para prescrever AR, $\mathbf{n}(\%)$ & $131(79,4)$ & $34(20,6)$ & $166(100,0)$ \\
Para efetuar rastreio, $\mathrm{n}(\%)$ & $116(70,3)$ & $49(29,7)$ & $166(100,0)$ \\
Para conhecer o perfil do utente, $\mathrm{n}(\%)$ & $45(27,3)$ & $120(72,7)$ & $166(100,0)$ \\
Para satisfazer a vontade do utente, $\mathrm{n}(\%)$ & $106(64,2)$ & $59(35,8)$ & $166(100,0)$ \\
\hline Para seguir a evidência científica, $\mathrm{n}(\%)$ & & & \\
\hline
\end{tabular}

AR: análises de rotina

Tabela 5 - Motivos para não prescrição de AR

\begin{tabular}{lrrr}
\hline & Sim & Não & Total \\
\hline Motivo para não prescrever AR, $\mathbf{n}(\%)$ & & & $157(100,0)$ \\
Preocupação com despesas em saúde, $\mathrm{n}(\%)$ & $49(31,4)$ & $107(68,6)$ & $157(100,0)$ \\
Por não considerar relevante, $\mathrm{n}(\%)$ & $62(39,7)$ & $94(60,3)$ & $157(100,0)$ \\
Porque não está de acordo com a evidência científica, $\mathrm{n}(\%)$ & $126(80,8)$ & $30(19,2)$ & $157(100,0)$ \\
\hline Outro, $\mathrm{n}(\%)$ & $16(10,2)$ & $141(89,8)$ & 1 \\
\hline
\end{tabular}

AR: análises de rotina 
indicado por estes médicos para a não requisição de análises. Em oposição, a maior parte dos médicos que responderam ao questionário pede AR com a intenção de realizar rastreio remetendo para a problemática da manutenção de cuidados de saúde não baseados na melhor evidência científica.

Destaca-se também o facto de haver discrepâncias no tipo de análises requisitadas, ou seja, nem todos os médicos que referem requisitar selecionaram o mesmo grupo de análises para avaliar os seus utentes, este facto demonstra que poderá não existir homogeneidade nos cuidados de saúde, variando conforme o médico assistente.

Não foi possível atingir o tamanho da amostra proposto inicialmente, no entanto, conseguiu-se demonstrar que existem diferenças estatisticamente significativas ao comparar a taxa de requisição entre grupos.

Apesar das limitações do estudo, como a dimensão inferior da amostra e o facto de ter sido apenas avaliado um conjunto limitado de análises, os resultados apresentados ajudam-nos a confirmar as diferenças existentes na prática de MGF em Portugal, nomeadamente no que diz respeito à requisição de AR por médicos de diferentes regiões, graus de especialização e idade. O estudo permitiu também sedimentar a noção de que é premente investir na promoção e literacia de saúde no sentido de melhor justificar, junto dos nossos utentes, a evicção de práticas não comprovadas pela evidência.

Atualmente somos confrontados com o envio de mensagens contraditórias, relativamente à requisição de meios complementares de diagnóstico, no que diz respeito aos serviços de saúde privados e públicos. Ao nível dos cuidados de saúde secundários, poderá existir uma prescrição desadequada de AR, quer dentro quer fora do âmbito da especialidade. São necessários estudos subsequentes para analisar se esta prescrição pode influenciar ou estar influenciada pela prática dos cuidados de saúde primários.

Seria ainda interessante estudar o impacto económico associado à requisição inapropriada de AR em Portugal, no contexto global da saúde e não apenas nos cuidados de saúde primários, principalmente atendendo ao panorama financeiro do Serviço Nacional de Saúde. ${ }^{20}$

\section{CONCLUSÃO}

Os nossos resultados sugerem que existe um pedido excessivo de análises de "rotina".

É, pois, imperativa a mudança cultural de uma perspetiva patogénica para salutogénica o que só será conseguido através de estratégias que permitam implementar mudança de pensamento aliada a literacia em saúde da sociedade atual, dado que o papel do médico de família, nesta alteração, é ínfimo. Para a mudança deste paradigma uma estratégia globalizada terá um impacto muito mais significativo do que ações individualizadas ao nível da Medicina Geral e Familiar.

O desafio é lançado, para a criação de estratégias de marketing social para mudar a cultura vigente no sentido de reduzir o risco público associado a sobre-utilização, sobrediagnóstico e sobretratamento que a realização inapropriada de exames acarreta. ${ }^{13}$

\section{OBSERVAÇÕES}

Uma parte do trabalho foi apresentada em 5 de novembro de 2018 no Congresso: $2^{\text {nd }}$ European Forum on Prevention and PrimaryCare (Porto), tendo recebido o prémio de melhor comunicação oral.

\section{AGRADECIMENTOS}

A António Vaz Carneiro e a Paula Broeiro.

\section{PROTEÇÃO DE PESSOAS E ANIMAIS}

Os autores declaram que os procedimentos seguidos estavam de acordo com os regulamentos estabelecidos pelos responsáveis da Comissão de Investigação Clínica e Ética e de acordo com a Declaração de Helsínquia da Associação Médica Mundial.

\section{CONFIDENCIALIDADE DOS DADOS}

Os autores declaram ter seguido os protocolos do seu centro de trabalho acerca da publicação de dados.

\section{CONFLITOS DE INTERESSE}

Os autores declaram que não há conflito de interesses na publicação deste artigo.

\section{FONTES DE FINANCIAMENTO}

Os autores declaram não ter recebido subsídios ou bolsas para a elaboração do artigo.

\section{REFERÊNCIAS}

1. World Organization of Family Doctors (WONCA). European definition of general practice family medicine [Internet]. $3^{\text {rd }}$ WONCA Europe; 2011. [consultado em 2018 nov 14]. Disponível em: https://www.woncaeurope. org/sites/default/files/documents/Definition\%203rd\%20ed\%202011\%20 with\%20revised\%20wonca\%20tree.pdf.

2. Rakel R, Rakel D. Textbook of family medicine. $9^{\text {th }}$ ed. Philadelphia: Saunders; 2015.

3. KrogsbøII LT, Jørgensen KJ, Larsen CG, Gøtzsche PC. General health checks in adults for reducing morbidity and mortality from disease: Cochrane systematic review and meta-analysis. BMJ. 2012;345:e7191.

4. Brodersen J. Overdiagnosis: an unrecognised and growing worldwide problem in healthcare. Zdr Varst. 2017;56:147-9.
5. Heleno B, Thomsen MF, Rodrigues DS, Jørgensen KJ, Brodersen J. Quantification of harms in cancer screening trials: literature review. BMJ. 2013;347:f5334.

6. Martins C, Azevedo LF, Ribeiro O, Sá L, Santos P, Couto L, et al. A population-based nationwide cross-sectional study on preventive health services utilization in Portugal-what services (and frequencies) are deemed necessary by patients? PLoS ONE 2013;8:e81256;

7. Martins C, Azevedo LF, Santos C, Sá L, Santos P, Couto M, et al. Preventive health services implemented by family physicians in Portugal-a cross-sectional study based on two clinical scenarios. BMJ Open. 2014;4:e005162.

8. Practitioners RAC of G. Guidelines for preventive activities in general 
practice. $8^{\text {th }}$ ed. South Melbourne: Vic. Royal Australian College of General Practitioners; 2012.

9. Brotons C, Björkelund C, Bulc M, Ciurana R, Godycki-Cwirko M, Jurgova $\mathrm{E}$, et al. Prevention and health promotion in clinical practice: the views of general practitioners in Europe. Prev Med. 2005;40:595-601.

10. Faulkner A, Reidy M, McGowan J. Should we abandon routine blood tests? BMJ. 2017;357:j2091.

11. Nocs.pt. Prescrição e determinação do hemograma. Normas de Orientação Clínica [Internet]. [consultado em 2018 out 02]. Disponível em: http://nocs.pt/prescricao-determinacao-hemograma/.

12. Nocs.pt. Indicações para Prescrição do lonograma. Normas de Orientação Clínica [Internet]. [consultado em 2018 out 02]. Disponível em: http://nocs.pt/prescricao-ionograma/.

13. Sá L, Ribeiro O, Azevedo LF, Couto L, Costa-Pereira A, Hespanhol A, et al. Patients' estimations of the importance of preventive health services: a nationwide, population-based cross-sectional study in Portugal. BMJ Open. 05 de 2016;6(10): e011755.

14. Nocs.pt. Dislipidemias no Adulto. Normas de Orientação Clínica. [Internet]. [consultado em 2018 out 02]. Disponível em: http://nocs.pt/ exames-avaliacao- dislipidemias-adulto/.

15. U.S. Preventive Services Task Force. Final update summary: lipid disorders in adults (cholesterol, dyslipidemia): screening July 2015. [consultado em 2018 out 14]. Disponivel em: https:// www.uspreventiveservicestaskforce.org/Page/Document/ UpdateSummaryFinal/lipid-disorders-in-adults-cholesteroldyslipidemia-screening.

16. American Diabetes Association. Standards of medical care in diabetes-2016: summary of revisions. Diabetes Care. 2016;39:S4-5.

17. Lopes da Mota $C$, Beça HP. Análise sumária de urina de "rotina": porquê e para quê?. Rev Port Med Geral Fam. 2013;29:244-8.

18. European Confederation of Laboratory Medicine. European urinalysis guidelines. Scand J Clin Lab Invest Suppl. 2000;60:1-96.

19. Misra S, Barth $\mathrm{JH}$. How good is the evidence base for test selection in clinical guidelines? Clin Chim Acta. 2014;432:27-32.

20. Entidade Reguladora da Saúde. Análise da sustentabilidade financeira do Serviço Nacional de Saúde. Setembro 2011. [consultado em 2018 out 26]. Disponível em: https://www.ers.pt/uploads/writer_file/document/36/ Relatorio_Sustentabilidade_do_SNS.pdf. 